\title{
Increased expression of stem cell markers in malignant melanoma
}

\author{
Walter M Klein ${ }^{1}$, Bryan $\mathrm{P} \mathrm{Wu}^{1}$, Shuping Zhao ${ }^{1}$, Hong $\mathrm{Wu}^{2}$, Andres JP Klein-Szanto ${ }^{2}$ and \\ Steven R Tahan ${ }^{1}$ \\ ${ }^{1}$ Department of Pathology, Beth Israel Deaconess Medical Center and Harvard Medical School, Boston, \\ MA, USA and ${ }^{2}$ Department of Pathology, Fox Chase Cancer Center, Philadelphia, PA, USA
}

\begin{abstract}
The potential role of stem cells in neoplasia is a subject of recent interest. Three markers of melanocytic stem cells have been described recently. CD166 is expressed on the surface of mesenchymal stem cells and has been found on human melanoma cell lines. CD133 is expressed on the surface of dermal-derived stem cells that are capable of differentiating into neural cells. Nestin is an intermediate filament expressed in the cytoplasm of neuroepithelial stem cells. In this study, we evaluate the expression of these markers and possible differences among banal nevi, primary melanoma, and metastastic melanoma. Tissue microarrays containing normal tissue and 226 melanocytic lesions (71 banal nevi, 71 in situ and invasive melanomas, and 84 metastatic melanomas) were studied by immunohistochemistry using monoclonal antibodies CD166, CD133, and nestin. A significantly greater percentage of melanomas (combined primary and metastatic) contained cells that expressed CD166 $(P=0.005), C D 133(P=0.003)$, and nestin $(P=0.03)$ than banal nevi. Only nestin showed a statistical difference when comparing primary and metastatic melanoma $(P=0.05)$. A stepwise increase in the proportion of lesions expressing all three markers was observed from banal nevi (2/19) to primary melanomas (8/17) to metastatic melanoma (19/28), $P=0.0005$. All cases of metastatic melanoma expressed at least one stem cell marker. The increased expression of CD166, CD133, and nestin in melanoma suggests that progression to malignant melanoma likely involves genetic pathways instrumental to stem cell biology and normal tissue development. Further studies and characterization of these pathways may also reveal new prognostic markers for a disease whose prognosis in advanced stages is dismal. Modern Pathology (2007) 20, 102-107. doi:10.1038/modpathol.3800720; published online 24 November 2006
\end{abstract}

Keywords: melanoma; stem cell; CD133; CD166; nestin

Melanoma is the sixth most common cancer in the United States and its incidence has been increasing over the past few decades. ${ }^{1}$ Despite improvements in overall survival, the prognosis for advanced-stage disease remains poor. ${ }^{2}$ Multivariate analyses have shown that the two most powerful histologic predictors for survival are tumor thickness and

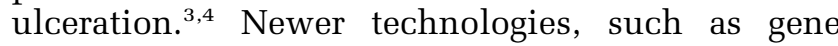
expression profiling and proteomics, are rapidly expanding our knowledge of melanoma cell biology. In addition, these promise to lead to the identification of new prognostic and therapeutic markers, possibly including stem cell markers.

The potential role of stem cells in neoplasia is a subject of recent interest. The cancer stem cell

Correspondence: Dr SR Tahan, MD, Department of Pathology East Campus, Beth Israel Deaconess Medical Center and Harvard Medical School, 330 Brookline Avenue, Boston, MA 02215, USA. E-mail: stahan@bidmc.harvard.edu

Received 23 June 2006; revised 3 October 2006; accepted 9 October 2006; published online 24 November 2006 hypothesis suggests that malignant tumors are comprised of both cancer stem cells, which have great proliferative potential, as well as more differentiated cancer cells, with limited proliferative potential. ${ }^{5,6}$ The existence of cancer stem cells has been established in many different malignancies including acute myeloid leukemia, breast cancer, and glioblastoma. ${ }^{7-10}$ Identification of cancer stem cells may help refine classification, diagnosis and treatment of these cancers and potentially others including melanoma.

Recently, three stem cell markers, that which are also expressed on melanocytes have been described. Activated leukocyte adhesion molecule (CD166) is a member of the immunoglobulin super family and is a type 1 transmembrane protein. CD166 is expressed on the surface of mesenchymal stem cells and has been found on human melanoma cell lines. ${ }^{11} \mathrm{In}$ addition, its expression correlates with tumor thickness in primary melanoma. ${ }^{12}$ CD133 (human prominin-1/AC133) is a transmembrane glycoprotein that is expressed on the hematopoietic stem 
cells, endothelial progenitors, and dermal-derived stem cells capable of differentiating into neural cells. ${ }^{13,14}$ Nestin is an intermediate filament expressed in the cytoplasm of neuroepithelial stem cells. ${ }^{15,16}$ Its expression has also been found in metastatic melanomas. ${ }^{17}$

This study was designed to assess whether the expression of these three stem cell markers identify differences in melanocytic cells comprising banal nevi, primary melanoma, and metastatic melanoma. More specifically, do primary melanomas and metastatic melanomas have an increased percentage of cells expressing stem cell markers relative to banal nevi; and if so, could these cells represent a 'cancer stem cell' population?

\section{Materials and methods}

Five micron sections of tissue microarrays containing normal tissue and 226 melanocytic lesions (71 banal nevi most of which were dermal nevi, 71 in situ and invasive melanomas, and 84 metastatic melanomas) were mounted on covalent slides. Monoclonal antibodies for CD166 (BD clone 3A6), CD133 (Miltenyi Biotec clone AC133), and nestin (Chemicon clone 10C2) were assayed using standard $\mathrm{ABC}$ immunohistochemical technique with red alkaline phosphatase substrate. Slides were reviewed by two pathologists. Positive staining was quantitatively interpreted as focal $(<15 \%$ of lesional cells) or diffuse ( $>15 \%$ of lesional cells). In addition, the intensity of staining was also determined subjectively as weak, moderate, and strong based on positive internal controls for each stain. Lesions, which were scored as focal weak, were interpreted as negative in the final data analysis. Fisher's exact test was used for group comparisons.

\section{Results}

All three immunohistochemical stains demonstrated cytoplasmic staining. Scattered positive staining for CD166 was observed in lymphocytes and fibroblasts. CD133 staining was also seen in a few lymphocytes, occasional endothelial cells, and other mesenchymal cells. Nestin expression was seen in the outer root sheath of mid-lower hair follicles and dermal mesenchymal cells. The results for the 226 melanocytic lesions are shown in Table 1.

Table 1 CD166, CD133, and Nestin staining of 226 melanocytic lesions

\begin{tabular}{lccc}
\hline & Nevus & $\begin{array}{c}\text { Primary } \\
\text { melanoma }\end{array}$ & $\begin{array}{c}\text { Metastatic } \\
\text { melanoma }\end{array}$ \\
\hline CD166 pos/total (\%) & $11 / 71(15)$ & $37 / 70(53)$ & $58 / 84(69)$ \\
CD133 pos/total (\%) & $12 / 71(17)$ & $28 / 71(39)$ & $39 / 84(46)$ \\
Nestin pos/total (\%) & $35 / 64(55)$ & $44 / 63(70)$ & $69 / 80(86)$
\end{tabular}

\section{CD166}

CD166 expression was identified in $11 / 71$ banal nevi, 37/70 primary melanomas, and 58/84 metastatic melanomas (Figure 1a-c). The majority of positive staining nevi demonstrated either diffuse
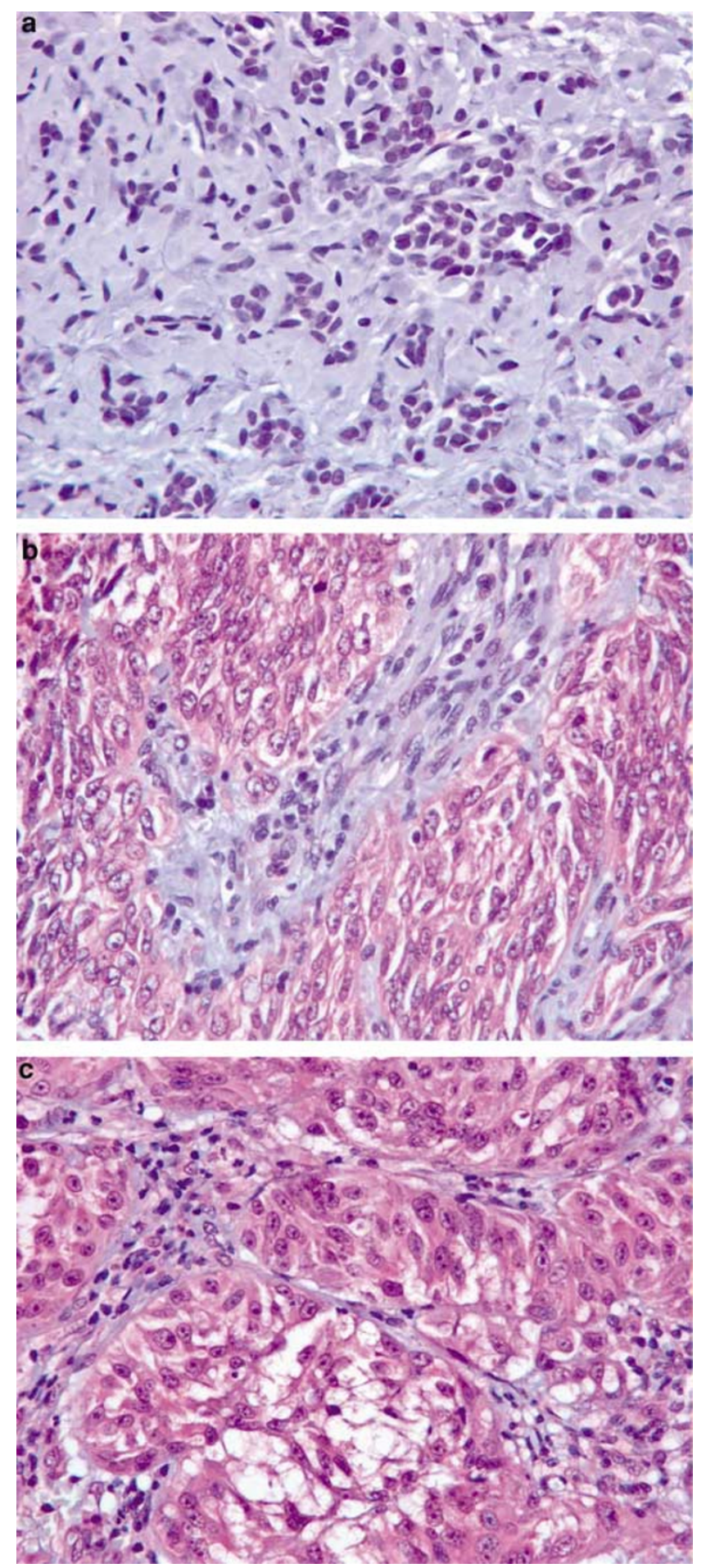

Figure 1 (a) Dermal nevus showing no staining for CD166. (b) Primary melanoma showing strong and diffuse staining for CD166. (c) Metastatic melanoma showing strong and diffuse staining for CD166. 
weak staining or focal staining (5-15\% of cells). Often staining was observed in the junctional component or in nevic nests in the upper papillary dermis. None of the nevi revealed diffuse strong staining. In contrast, among positive staining cases in primary melanomas and metastatic melanomas, staining tended to be diffuse with a spectrum ranging from weak to moderate to strong (33/37 and 55/58, respectively). Focal staining, regardless of intensity, was infrequent in primary melanomas and metastatic melanomas $(4 / 37$ and $3 / 58$, respectively).

\section{CD133}

CD133 expression was observed in 12/71 banal nevi, $28 / 71$ primary melanomas, and $39 / 84$ metastatic melanomas (Figure 2a-c). The majority of positive staining nevi demonstrated focal moderate staining (5-15\% of cells), and occasionally moderate diffuse staining. None of the nevi revealed diffuse strong staining. Among positive staining cases in primary melanomas and metastatic melanomas, staining tended to be diffuse with a spectrum ranging from weak to moderate-to-strong $(24 / 28$ and $34 / 39$, respectively). Focal staining, regardless of intensity, was infrequent in primary melanomas and metastatic melanomas (4/28 and 5/39, respectively).

\section{Nestin}

Nestin expression was identified in $35 / 64$ banal nevi, 44/63 primary melanomas, and 69/80 metastatic melanomas (Figure 3a-c). Focal moderate and weak diffuse staining was most often observed in nevi. None of the nevi demonstrated diffuse strong staining. In contrast, diffuse staining of moderate to strong intensity was frequently seen in primary melanomas and metastatic melanomas (27/44 and 43/69, respectively). Focal staining, regardless of intensity, was infrequent in primary and metastatic melanomas (5/44 and 6/69, respectively).

\section{Statistical Analysis}

A significantly greater percentage of melanomas (combined primary and metastatic) contained cells expressing CD166 $(P=<0.0001), \quad$ CD133 $(P=<0.0001)$, and nestin $(P=0.0007)$ than banal nevi. Only nestin showed a statistical difference when comparing primary and metastatic melanoma $(P=0.025)$.

A stepwise increase in the proportion of lesions expressing all the three markers was observed from banal nevi $(5 / 64)$ to primary melanomas $(18 / 63)$ to metastatic melanoma (28/79), $P=0.0072$. Similarly, there was a stepwise decrease in the proportion of lesions demonstrating negative staining for all three markers (banal nevi 23/64, primary melanoma 8/63,
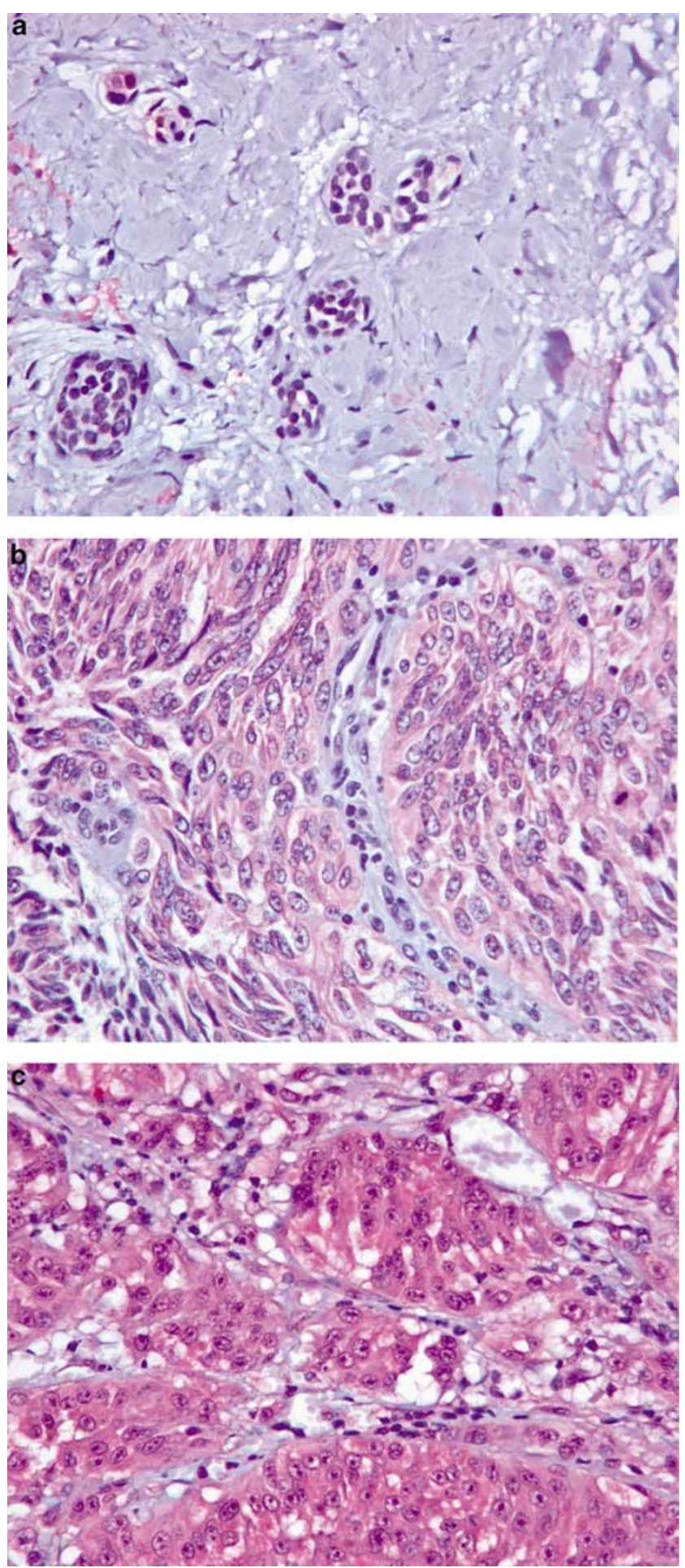

Figure 2 (a) Dermal nevus showing focal staining for CD133. (b) Primary melanoma showing strong and diffuse staining for CD133. (c) Metastatic melanoma showing strong and diffuse staining for CD133.

and metastatic melanoma 4/79, $P=0.0002$. Most cases of metastatic melanoma (75/79) expressed at least one stem cell marker. 

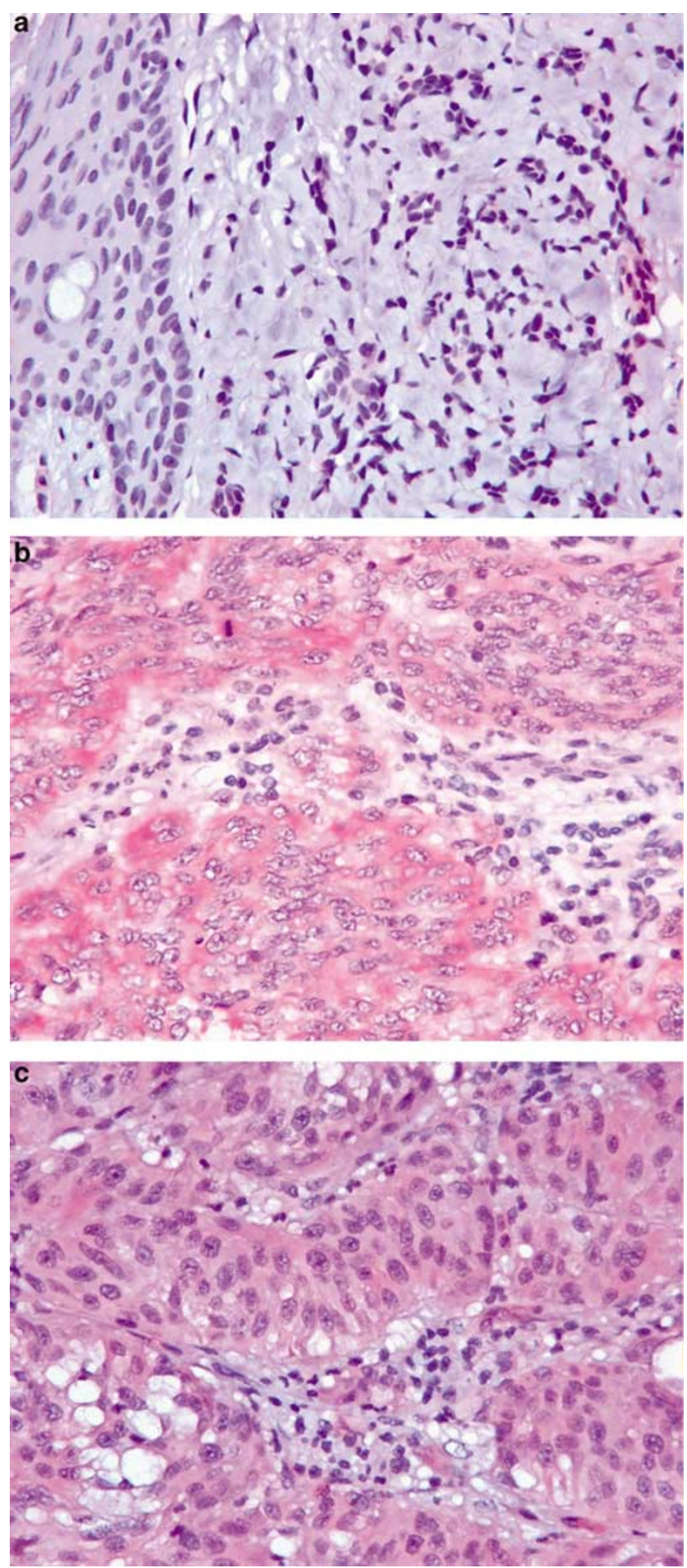

Figure 3 (a) Dermal nevus showing no staining for nestin. (b) Primary melanoma showing strong and diffuse staining for nestin. (c) Metastatic melanoma showing strong and diffuse staining for nestin.

\section{Discussion}

The classic hypothesis is that melanoma arises from a mature, differentiated melanocyte that has undergone a series of genetic alterations of tumor suppressor genes and oncogenes, such as CDKN2A, PTEN, TP53, RAS, and MYC. ${ }^{18}$ However, there is increasing evidence that cancer may arise from transformed stem cells that are able to self-renew, differentiate into multiple cell lineages, and drive continuous growth. The existence of cancer stem cells has been established in many different malignancies including acute myeloid leukemia, breast cancer, and glioblastoma. ${ }^{7-10}$

The bulge region of the murine hair follicle contains multipotent stem cells that have the potential to generate all the epithelial lineages of skin. ${ }^{19}$ In addition, it appears that other cells in the bulge region, thought to be of neural crest origin, can produce cells expressing neuronal, glial, smooth muscle, and melanocyte markers. ${ }^{20}$ Melanocytic stem cells have been identified in bulge area of the murine hair follicle. ${ }^{21}$ In humans, experiments with hair cycling and vitiligo have shown new melanocytes to appear, thus supporting the concept that melanocytes in human skin are replenished from a reservoir of stem cells. ${ }^{21,22}$ These melanocytic stem cells may have a role in the pathogenesis of banal nevi and melanoma.

CD166, a member of the immunoglobulin super family and a type 1 transmembrane glycoprotein, is involved in cell adhesion and cytoskeletal anchoring. It has been detected in a subset of cells in a variety of human tissues (thought to be pluripotent stem cells) including epithelia, lymphoid and myeloid cells, fibroblasts, neurons, hepatocytes, and pancreas acinar and islet cells. Its temporal and spatial expression has been documented in animals, and correlates with different stages of biological development including neural development, branching organ development, hematopoiesis, and immune response. ${ }^{11}$

A previous study demonstrated that increased CD166 expression was seen in melanomas with a Breslow depth $\geq 1.5 \mathrm{~mm}$ and metastatic melanomas, whereas thinner melanomas and benign nevi showed less expression. ${ }^{12}$ Similar to our findings, nevi, when positive, showed staining limited to the junctional component or nevic nests in the superficial papillary dermis. The authors speculate that CD166 plays an important role in melanoma cell invasion and melanoma progression.

CD133, in addition to being normally expressed on the surface of hematopoeitic stem cells, endothelial progenitors, and fetal brain, has recently been used to identify a subset of dermal-derived cells capable of differentiating into neurons in vitro as well as endothelium and astrocytes in vivo. ${ }^{13,14}$ CD133 is also expressed by acute and chronic myeloid leukemias as well as lymphoblastic lymphoma. ${ }^{23}$ In a xenograft study of gliobastomas, a small fraction of CD133-positive cells were capable of tumor initiation closely resembling the patient's primary tumor, whereas CD133-negative cells were incapable of producing tumors even when grafting large numbers of tumor cells. ${ }^{24}$ More recently, 
ABCB5, a novel drug transporter and chemoresistance mediator, was coexpressed with CD133 in distinct tumor subpopulations in both cultured melanoma cells and clinical melanoma tumors. ${ }^{25}$ Tumor growth and metastasis driven by chemoresistant cancer stem cells might explain the failure of existing therapies to consistently eradicate solid tumors. ${ }^{6}$

Nestin, first identified in the cytoplasm of neuroepithelial stem cells, is also expressed in migrating and proliferating cells during embryogenesis and in various adult tissues undergoing regeneration, such as the central nervous system, liver, pancreas, and gastrointestinal tract. ${ }^{26}$ Nestin expression has been found in glioblastoma multiforme, primitive neuroectodermal tumors of the CNS, and metastatic melanoma. ${ }^{17,27}$ Its expression seems to correlate with the high proliferative and migrational activity of these tumors.

A recent study using metastatic melanoma cell lines identified cells with heterogenous morphology and antigenic characteristics including a smaller cell population that labeled with nestin. In addition, this subset of cells had a slower proliferative rate, but was able to drive cellular expansion in culture. This observation led the authors to speculate that melanoma may be a tumor based on a mutant stem cell, attempting to undergo normal developmental processes. ${ }^{28}$

Another recent study with metastatic melanoma cell lines identified a population of melanoma cells that had the ability to grown in spheres (a common growth characteristic of stem cells), self-renew, and differentiate into melanogenic, adipogenic, chrondrogenic, and osteogenic lineages. ${ }^{29}$ In this study, a subpopulation of these cells that expressed the hematopoietic marker CD20 tended to form larger spheres and had more potential for mesenchymal differentiation. The authors report identifying individual $\mathrm{CD}_{20}{ }^{+}$tumor cells in metastatic melanomas (data not shown). In addition, in a separate study, CD20 has been identified by gene expression profiling as one of the top 22 genes that mark aggressive melanoma. ${ }^{30}$ Correlation of differentiation ability and tumorigenicity by comparing $\mathrm{CD} 20^{+}$ with $\mathrm{CD} 20^{-}$fractions has not yet been demonstrated; however, this intriguing observation identifies a potentially important tumorigenic subpopulation of melanoma cells with stem cell properties.

In this study, we have shown that relative to banal nevi, both primary melanoma and metastatic melanoma show an increased expression of three stem cell markers. Self-limited mutational events of melanocytic stem cells may account for the formation of banal nevi whereas additional mutations may lead to the evolution of a malignant stem cell with the decreased ability to produce differentiated mature nevomelanocytes. This could account for the increased staining observed in primary and metastatic melanoma with respect to both percentage of cells and staining intensity.
The increased expression of CD166, CD133, and nestin in melanoma suggests that progression to malignant melanoma likely involves genetic pathways instrumental to stem cell biology and normal tissue development. A genetically defective melanocytic stem cell could account for the formation of both melanocytic nevi and melanoma. Further studies and characterization of these pathways may also reveal new prognostic markers and targeted treatment strategies for a disease whose prognosis is dismal in advanced stages.

\section{Acknowledgement}

Supported by Division of Dermatopathology, Beth Israel Deaconess Medical Center.

\section{References}

1 Jemal A, Murray T, Ward E, et al. Cancer statistics, 2005. CA Cancer J Clin 2005;55:10-30.

2 Rigel DS, Friedman RJ, Kopf AW. The incidence of malignant melanoma in the United States: issues as we approach the 21st century. J Am Acad Dermatol 1996; 34:839-847.

3 Balch CM, Soong SJ, Gershenwald JE, et al. Prognostic factors analysis of 17, 600 melanoma patients: validation of the American Joint Committee on Cancer melanoma staging system. J Clin Oncol 2001;19: 3622-3634.

4 Eigentler TK, Buettner PG, Leiter U, et al. Impact of ulceration in stages I to III cutaneous melanoma as staged by the American Joint Committee on Cancer Staging System: an analysis of the German Central Malignant Melanoma Registry. J Clin Oncol 2004;22: 4376-4383.

5 Hamburger AW, Salmon SE. Primary bioassay of human tumor stem cells. Science 1977;197:461-463.

6 Reya T, Morrison SJ, Clarke MF, et al. Stem cells, cancer, and cancer stem cells. Nature 2001;414: 105-111.

7 Al-Hajj M, Wicha MS, Benito-Hernandez A, et al. Prospective identification of tumorigenic breast cancer cells. Proc Natl Acad Sci USA 2003;100:3983-3988.

8 Bonnet D, Dick JE. Human acute myeloid leukemia is organized as a hierarchy that originates from a primitive hematopoietic cell. Nat Med 1997;3:730-737.

9 Lapidot T, Sirard C, Vormoor J, et al. A cell initiating human acute myeloid leukaemia after transplantation into SCID mice. Nature 1994;367:645-648.

10 Singh SK, Clarke ID, Terasaki M, et al. Identification of a cancer stem cell in human brain tumors. Cancer Res 2003;63:5821-5828.

11 Swart GW, Lunter PC, Kilsdonk JW, et al. Activated leukocyte cell adhesion molecule (ALCAM/CD166): signaling at the divide of melanoma cell clustering and cell migration? Cancer Metastasis Rev 2005;24: 223-236.

12 van Kempen LC, van den Oord JJ, van Muijen GN, et al. Activated leukocyte cell adhesion molecule/CD166, a marker of tumor progression in primary malignant melanoma of the skin. Am J Pathol 2000;156:769-774. 
13 Belicchi M, Pisati F, Lopa R, et al. Human skin-derived stem cells migrate throughout forebrain and differentiate into astrocytes after injection into adult mouse brain. J Neurosci Res 2004;77:475-486.

14 Shmelkov SV, St Clair R, Lyden D, et al. AC133/CD133/ Prominin-1. Int J Biochem Cell Biol 2005;37:715-719.

15 Dahlstrand J, Zimmerman LB, McKay RD, et al. Characterization of the human nestin gene reveals a close evolutionary relationship to neurofilaments. J Cell Sci 1992;103(Part 2):589-597.

16 Lendahl U, Zimmerman LB, McKay RD. CNS stem cells express a new class of intermediate filament protein. Cell 1990;60:585-595.

17 Florenes VA, Holm R, Myklebost O, et al. Expression of the neuroectodermal intermediate filament nestin in human melanomas. Cancer Res 1994;54:354-356.

18 Rodolfo M, Daniotti M, Vallacchi V. Genetic progression of metastatic melanoma. Cancer Lett 2004;214: 133-147.

19 Oshima H, Rochat A, Kedzia C, et al. Morphogenesis and renewal of hair follicles from adult multipotent stem cells. Cell 2001;104:233-245.

20 Amoh Y, Li L, Katsuoka K, et al. Multipotent nestinpositive, keratin-negative hair-follicle bulge stem cells can form neurons. Proc Natl Acad Sci USA 2005; 102:5530-5534.

21 Nishimura EK, Jordan SA, Oshima H, et al. Dominant role of the niche in melanocyte stem-cell fate determination. Nature 2002;416:854-860.
$22 \mathrm{Yu}$ HS. Melanocyte destruction and repigmentation in vitiligo: a model for nerve cell damage and regrowth. J Biomed Sci 2002;9:564-573.

23 Bhatia M. AC133 expression in human stem cells. Leukemia 2001;15:1685-1688.

24 Singh SK, Hawkins C, Clarke ID, et al. Identification of human brain tumour initiating cells. Nature 2004;432: 396-401.

25 Frank NY, Margaryan A, Huang Y, et al. ABCB5mediated doxorubicin transport and chemoresistance in human malignant melanoma. Cancer Res 2005;65: 4320-4333.

26 Wiese C, Rolletschek A, Kania G, et al. Nestin expression-a property of multi-lineage progenitor cells? Cell Mol Life Sci 2004;61:2510-2522.

27 Tohyama T, Lee VM, Rorke LB, et al. Nestin expression in embryonic human neuroepithelium and in human neuroepithelial tumor cells. Lab Invest 1992;66: 303-313.

28 Grichnik JM, Burch JA, Schulteis RD, et al. Melanoma, a tumor based on a mutant stem cell? J Invest Dermatol 2006;126:142-153.

29 Fang D, Nguyen TK, Leishear K, et al. A tumorigenic subpopulation with stem cell properties in melanomas. Cancer Res 2005;65:9328-9337.

30 Bittner M, Meltzer P, Chen Y, et al. Molecular classification of cutaneous malignant melanoma by gene expression profiling. Nature 2000;406: 536-540. 